\title{
Динамика заболеваемости в периоды до и после принятия мер по недопущению распространения новой коронавирусной инфекции и смертность от новой коронавирусной инфекции в разных субъектах Российской Федерации в период с 01/09/2021 по 15/01/2022
}

Эдвард Михайлович Гольдштейн ${ }^{1}$

1. Гарвардская школа общественного здравоохранения, Бостон, США/Москва, Россия edmigo3@gmail.com

\section{Аннотация}

Актуальность: Меры по недопущению распространения новой коронавирусной инфекции могут иметь значительное влияние на динамику заболеваемости и смертности. Например, после указа Президента об установлении нерабочих дней в период с 30 октября по 7 ноября 2021 г., и сопутствующих ограничительных мер, принятых главами/правительствами ряда субъектов Российской Федерации, пик заболеваемости по Российской Федерации в целом был зафиксирован 6 ноября 2021г. Мы изучили публикации о мерах по недопущению распространения новой коронавирусной инфекции (ограничительных мерах), принятых главами/правительствами разных субъектов Российской Федерации осенью 2021 года, и связь между моментом принятия первых популяционно значимых ограничительных мер и динамикой заболеваемости и смертности от новой коронавирусной инфекции в разных субъектах Российской Федерации в период с 01/09/2021 по 15/01/2022. Методы: Данные о ежедневной заболеваемости и смертности от новой коронавирусной инфекции в разных субъектах Российской Федерации были получены с сайта стопкоронавирус.рф. Мы установили хронологию принятия ограничительных мер осенью 2021 года в 82/85 субъектах Российской Федерации. Мы оценили количество дней с момента принятия первых 
популяционно значимых ограничительных мер до пика эпидемии, а также длину периода роста заболеваемости до момента принятия первых популяционно значимых ограничительных мер. Результаты: В среднем по 82 субъектам Российской Федерации, количество дней с момента принятия первых, популяционно значимых ограничительных мер до пика заболеваемости было 29.9 дней, а длина периода роста заболеваемости до принятия соответствующих ограничительных мер была 20.6 дней. Корреляция между недельным уровнем смертности от новой коронавирусной инфекции в период роста заболеваемости до принятия ограничительных мер и смертностью от новой коронавирусной инфекции на 100,000 человек в период с 10/10/2021 по 15/01/2022 для 82 регионов Российской Федерации была 0.73 (95\% Ди $(0.60,0.81))$. Корреляция между длиной периода роста заболеваемости до принятия ограничительных мер и смертностью от новой коронавирусной инфекции на 100,000 человек в период с 10/10/2021 по 15/01/2022 была $0.23(0.01,0.42)$. Корреляция между длиной периода роста заболеваемости до принятия ограничительных мер и избыточной смертностью от всех причин в период с сентября по октябрь 2021 года для 82 регионов Российской Федерации была 0.32 (0.11,0.50). Выводы: Своевременное принятие ограничительных является одним из факторов, влияющих на смертность от новой коронавирусной инфекции. Введение дополнительных критериев для принятия мер по недопущению распространения новой коронавирусной инфекции -- например, принятие ограничительных мер после двух недель роста заболеваемости при достаточно высоком уровне смертности - может способствовать уменьшению последующей заболеваемости и смертности от новой коронавирусной инфекции. Также, наши результаты говорят о влиянии совокупности различных ограничительных мер на последующее распространение новой коронавирусной инфекции.

\section{Введение}

Эпидемия новой коронавирусной инфекции в период с сентября 2021 года по декабрь 2021 года в Российской Федерации была связана с высокими уровнями 
заболеваемости и смертности от новой коронавирусной инфекции [1,2]. Также, только для части смертей, связанных с новой коронавирусной инфекцией, новая коронавирусная инфекция является лабораторно подтвержденной. Уровень смертности от всех причин заметно повышается в периоды активной циркуляции новой коронавирусной инфекции в разных странах, включая Российскую Федерацию $[3,4]$. Рост уровня смертности от всех причин в период эпидемии новой коронавирусной инфекции в Российской Федерации осенью 2021 года наблюдался уже в октябре 2021 года [5,6].

Исследования в ряде стран показали, что меры по недопущению распространения новой коронавирусной инфекции имеют значительное влияние на динамику заболеваемости [7]. Осенью 2021 года различные меры по недопущению распространения новой коронавирусной инфекции были введены в разных субъектах Российской Федерации. После указа Президента от 20/10/2021 об установлении нерабочих дней в период с 30 октября по 7 ноября 2021 г. [8], и сопутствующих ограничительных мер, принятых главами/правительствами ряда субъектов Российской Федерации, пик заболеваемости по Российской Федерации в целом был зафиксирован 6 ноября 2021г [2]. В этой статье мы использовали данные Роспотребнадзора $[1,2]$ о ежедневной заболеваемости и смертности от новой коронавирусной инфекции в разных субъектах Российской Федерации для того, чтобы оценить динамику заболеваемости и уровень смертности от новой коронавирусной инфекции до момента принятия первых популяционно значимых мер по недопущению распространения новой коронавирусной инфекции осенью 2021 года, и связь между этими величинами и смертностью от новой коронавирусной инфекции в период с 10/10/2021 по 15/01/2022 в разных субъектах Российской Федерации. Отметим, что в 81/82 регионах Российской Федерации, включенных в наше исследование, принятие первых популяционно значимых мер по недопущению распространения новой коронавирусной инфекции предшествовало вступлению в силу указа Президента о нерабочих днях [8]. Оценки такого типа могут быть полезными при выработке критериев для введения мер по недопущению распространения новой коронавирусной инфекции в целях 
уменьшения последующей заболеваемости и смертности о новой коронавирусной инфекции.

\section{Методы}

\section{Данные}

Данные Роспотребнадзора о ежедневном количестве диагностированных заболеваний и смертей от новой коронавирусной инфекции в разных субъектах Российской Федерации доступны в [1,2]. Информация о постановлениях/решениях/указах, принятых главами/правительствами разных субъектов Российской Федерации осенью 2021 года доступна в порталах правовых документов $[9,10]$. Информация о численности населения в разных субъектах Российской Федерации доступна в [11].

\section{Принятие мер по недопущению распространения новой коронавирусной инфекции (ограничительных мер)}

Мы установили хронологию принятия ограничительных мер осенью 2021 года в 82/85 субъектах Российской Федерации. В каждом из этих 82 субъектов Российской Федерации, мы определили момент (день) принятия первых популяционно значимых ограничительных мер. Популяционно значимыми считались такие меры как ограничения на проведение развлекательных/культурных мероприятий, меры по социальному дистанцированию в местах общественного питания и в других общественных местах, использование QR кода и других подтверждений статуса заболевания/вакцинации при посещении общественных мест/мероприятий, и т.д. Меры, направленные на специальные типы учреждений (такие как врачебнофизкультурные диспансеры и др.) не считались сами по себе популяционно значимыми для распространения новой коронавирусной инфекции в населении, и введение только таких мер не расценивалось как момент принятия первых популяционно значимых ограничительных мер. 


\section{Период роста заболеваемости и другие параметры}

Период роста заболеваемости определялся как последовательность идущих один за другим трехдневных интервалов до момента принятия ограничительных мер (т.е. интервалов [д,д-2], [д-3,д-5], [д-7,д-9], и т.д., где “д” это день, в который период роста заканчивался), для которых количество заболеваний в каждый трехдневный интервал превышало количество заболеваний в предыдущий трехдневный интервал. Последний из этих интервалов (для которого заболеваемость не увеличилась по сравнению с предыдущим трехдневным интервалом) не включался в период роста заболеваемости; при этом, мы включали 0,1 или 2 последних дня из этого трехдневного периода в период роста заболеваемости в зависимости от динамики роста заболеваемости в этот последний трехдневный период. Для каждого субъекта Российской Федерации мы определили период роста заболеваемости до дня П принятия популяционно значимых мер по недопущению распространения новой коронавирусной инфекции как один из трех периодов роста, для которых день П, П-1 или П-2 были последними днями, в зависимости от того, длина какого из этих 3-х периодов роста была максимальной.

Прирост заболеваемости в период роста заболеваемости определялся как отношение между заболеваемостью в последний (в конце периода роста заболеваемости) трехдневный интервал, и первый (в начале периода роста заболеваемости) трехдневный интервал. Средний ежедневный прирост заболеваемости в период роста заболеваемости перед принятием ограничительных мер определялся как темп (экспоненциального) роста, который дает наблюдаемый прирост заболеваемости за время, равное длине периода роста заболеваемости перед принятием ограничительных мер.

Недельный уровень смертности в день д определялась как смертность от новой коронавирусной инфекции в предшествующий семидневный период [д-6,д] на 100,000 человек. Недельный уровень смертности в период роста заболеваемости перед принятием ограничительных мер определяется как средний недельный уровень смертности для дней, входящих в период роста. Отметим, что мы 
используем недельный уровень смертности потому, что он подвержен меньшим колебаниям по сравнению с ежедневным уровнем смертности.

Избыточная смертность от всех причин в определенный период в 2021 году определялась как смертность от всех причин на 100,000 человек в данный период в 2021 году (данные о количестве смертей от всех причин по октябрь 2021 года доступны в [5]), минус среднее значение между смертностью от всех причин в тот же период в 2018 и 2019 году.

Пик заболеваемости в каждом субъекте Российской Федерации определялся как день, начиная с 1 сентября 2021 года, в который (диагностированная) заболеваемость, усредненная по трехдневным периодам, была максимальной. Отметим, что в каждом из 82 регионов, включенных в исследование, пик заболеваемости наступал после момента принятия ограничительных мер.

\section{Результаты исследования}

Хронология мер по недопущению распространения новой коронавирусной инфекции (ограничительных мер) осенью 2021 года была установлена в 82/85 субъектах Российской Федерации. В Таблице 1 представлены различные величины, связанные с динамикой заболеваемости и смертности от новой коронавирусной инфекции и моментом принятия первых популяционно значимых мер по недопущению распространения новой коронавирусной инфекции (Методы) в 82 субъектах Российской Федерации. Отметим, что в 81/82 регионах Российской Федерации, включенных в наше исследование, принятие первых популяционно значимых мер по недопущению распространения новой коронавирусной инфекции предшествовало вступлению в силу указа Президента о нерабочих днях [8]. 


\begin{tabular}{|c|c|}
\hline $\begin{array}{l}\text { Среднее количество дней с момента принятия ограничительных } \\
\text { мер до пика заболеваемости }\end{array}$ & 29.9 дней \\
\hline $\begin{array}{l}\text { Средняя длина периода роста заболеваемости перед принятием } \\
\text { ограничительных мер }\end{array}$ & 20.6 дней \\
\hline $\begin{array}{l}\text { Средний недельный уровень смертности в период роста } \\
\text { заболеваемости перед принятием ограничительных мер }\end{array}$ & $\begin{array}{l}4.16 \text { на } 100,00 \\
\text { человек }\end{array}$ \\
\hline $\begin{array}{l}\text { Средний ежедневный прирост заболеваемости в период роста } \\
\text { заболеваемости перед принятием ограничительных мер }\end{array}$ & $1.74 \%$ \\
\hline $\begin{array}{l}\text { Средний момент принятия первых популяционно значимых } \\
\text { ограничительных мер осенью } 2021 \text { года }\end{array}$ & $\begin{array}{l}\text { 13-14 октября } \\
2021 \text { года }\end{array}$ \\
\hline
\end{tabular}

Таблица 1: Различные величины, связанные с динамикой заболеваемости и смертности от новой коронавирусной инфекции и моментом принятия первых популяционно значимых мер по недопущению распространения новой коронавирусной инфекции в 82 субъектах Российской Федерации.

В Таблице 2 представлены оценки корреляции между различными величинами, связанными с динамикой заболеваемости и смертности от новой коронавирусной инфекции, и принятием первых популяционно значимых ограничительных мер для 82 регионов Российской Федерации. Отметим, что мы оцениваем смертность от новой коронавирусной инфекции по 15/01/2022 в связи с определенных промежутком времени между диагнозом новой коронавирусной инфекции и последующей смертью (в исследовании [12], этот промежуток времени был оценен в среднем в 18.1 дней). Корреляция между недельным уровнем смертности в период роста заболеваемости до принятия ограничительных мер и уровнем смертности от новой коронавирусной инфекции в период с 10/10/2021 по 15/01/2022 для 82 регионов была 0.73 (95\%ДИ $(0.60,0.81))$. Корреляция между длиной периода роста заболеваемости до принятия ограничительных мер и смертностью от новой коронавирусной инфекции на 100,000 человек в период с 10/10/2021 по 15/01/2022 была $0.23(0.01,0.42)$. Корреляция между длиной периода роста заболеваемости до 
принятия ограничительных мер и избыточной смертностью от всех причин в период с сентября по октябрь 2021 года для 82 регионов Российской Федерации была $0.32(0.11,0.50)$. Наши результаты (Таблица 1 и 2) говорят о том, что в ряде регионов Российской Федерации принятию ограничительных мер осенью 2021 года предшествовал относительно длительный период медленного роста заболеваемости, а недельный уровень смертности в соответствующий период роста заболеваемости, а также длина этого периода роста заболеваемости были связаны со смертностью на 100,000 человек в период с 10/10/2021 по 15/01/2022.

Оценки корреляции между днем принятия первых популяционно значимых ограничительных мер и другими величинами (последняя строка в Таблице 2) говорят о том, что в регионах Российской Федерации, в которых ограничительные меры были приняты относительно поздно, принятие мер происходило после относительно длительного периода роста заболеваемости, и при относительно высоком темпе роста заболеваемости, но впоследствии, количество дней с момента принятия ограничительных мер до пика заболеваемости в этих регионах было ниже, чем в среднем для 82 регионов Российской Федерации. Эти результаты могут быть связаны с тем, что в регионах Российской Федерации, в которых ограничительные меры были введены позже, совокупность этих ограничительных мер и установления нерабочих дней начиная с 30 октября 2021 года после указа Президента [8] стала причиной более короткого периода между моментом принятия ограничительных мер главами/правительствами этих регионов и моментом пика заболеваемости по сравнению с регионами, где ограничительные меры были введены раньше.

Оценки корреляции между количеством дней с момента принятия первых популяционно значимых ограничительных мер до пика заболеваемости и другими величинами (3-й столбец в Таблице 2) говорят о том, что в регионах, в которых ограничительные меры были приняты после относительно короткого периода роста заболеваемости, или при относительно низком темпе роста заболеваемости, количество дней с момента принятия ограничительных мер до пика заболеваемости было выше, чем в среднем для 82 регионов Российской Федерации. Это может быть 
связанно с постепенностью введения дополнительных ограничительных мер в соответствующих регионах (Заключение).

\begin{tabular}{|c|c|c|c|c|}
\hline & $\begin{array}{l}\text { Смертность от новой } \\
\text { коронавирусной } \\
\text { инфекции на } \\
100,000 \text { человек в } \\
\text { период с } 10 / 10 / 2021 \\
\text { по 15/01/2022 }\end{array}$ & $\begin{array}{l}\text { Количество дней } \\
\text { с момента } \\
\text { принятия } \\
\text { ограничительных } \\
\text { мер до пика } \\
\text { заболеваемости }\end{array}$ & $\begin{array}{l}\text { Длина периода } \\
\text { роста } \\
\text { заболеваемости } \\
\text { перед принятием } \\
\text { ограничительных } \\
\text { мер }\end{array}$ & $\begin{array}{l}\text { Ежедневный прирост } \\
\text { заболеваемости в } \\
\text { период роста } \\
\text { заболеваемости перед } \\
\text { принятием } \\
\text { ограничительных мер }\end{array}$ \\
\hline $\begin{array}{l}\text { Количество дней с } \\
\text { момента принятия } \\
\text { ограничительных мер } \\
\text { до пика заболеваемости }\end{array}$ & $\begin{array}{l}-0.03 \\
(-0.24,0.19)\end{array}$ & & & \\
\hline $\begin{array}{l}\text { Длина периода роста } \\
\text { заболеваемости перед } \\
\text { принятием } \\
\text { ограничительных мер }\end{array}$ & $\begin{array}{l}0.23 \\
(0.01,0.42)\end{array}$ & $\begin{array}{l}-0.36 \\
(-0.54,-0.16)\end{array}$ & & \\
\hline $\begin{array}{l}\text { Ежедневный прирост } \\
\text { заболеваемости в } \\
\text { период роста } \\
\text { заболеваемости перед } \\
\text { принятием } \\
\text { ограничительных мер }\end{array}$ & $\begin{array}{l}0.08 \\
(-0.14,0.29)\end{array}$ & $\begin{array}{l}-0.34 \\
(-0.52,-0.13)\end{array}$ & $\begin{array}{l}-0.09 \\
(-0.30,0.13)\end{array}$ & \\
\hline $\begin{array}{l}\text { Недельный уровень } \\
\text { смертности в период } \\
\text { роста заболеваемости } \\
\text { перед принятием } \\
\text { ограничительных мер }\end{array}$ & $\begin{array}{l}0.73 \\
(0.60,0.81)\end{array}$ & $\begin{array}{l}-0.06 \\
(-0.27,0.16)\end{array}$ & $\begin{array}{l}0.09 \\
(-0.13,0.30)\end{array}$ & $\begin{array}{l}-0.04 \\
(-0.25,0.18)\end{array}$ \\
\hline $\begin{array}{l}\text { Момент принятия } \\
\text { первых популяционно } \\
\text { значимых } \\
\text { ограничительных мер }\end{array}$ & $\begin{array}{l}-0.09 \\
(-0.30,0.13)\end{array}$ & $\begin{array}{l}-0.68 \\
(-0.79,-0.55)\end{array}$ & $\begin{array}{l}0.26 \\
(0.04,0.45)\end{array}$ & $\begin{array}{l}0.27 \\
(0.05,0.46)\end{array}$ \\
\hline
\end{tabular}


Таблица 2: Корреляция между различными величинами, связанными с заболеваемостью и смертностью от новой коронавирусной инфекции и моментом принятия первых популяционно значимых мер по недопущению распространения новой коронавирусной инфекции для 82 субъектов Российской Федерации.

\section{Заключение}

Осенью 2021 года циркуляция новой коронавирусной в Российской Федерации была связана с высокими уровнями заболеваемости и смертности от новой коронавирусной инфекции [1,2]. Также, различные меры по недопущению распространения новой коронавирусной инфекции были введены в разных субъектах Российской Федерации, включая указ Президента об установлении нерабочих дней в период с 30 октября по 7 ноября 2021 г. [8]. Исследования в ряде стран показали, что меры по недопущению распространения новой коронавирусной инфекции имеют значительное влияние на ход эпидемии [7]. В этой статье мы оценили динамику заболеваемости до момента принятия первых популяционно значимых мер по недопущению распространения новой коронавирусной инфекции осенью 2021 года (такие меры были приняты до вступления в силу указа Президента о нерабочих днях в 81/82 субъектах Российской Федерации, включенных в наше исследование), количество дней с момента принятия соответствующих мер до пика заболеваемости, и связь между этими параметрами и смертностью от новой коронавирусной инфекции в разных субъектах Российской Федерации. Мы нашли, что в ряде регионов Российской Федерации принятию ограничительных мер осенью 2021 года предшествовал относительно длительный период медленного роста заболеваемости, а недельный уровень смертности в соответствующий период роста заболеваемости, и длина этого периода роста заболеваемости были связаны со смертностью от новой коронавирусной инфекции на 100,000 человек в период с 10/10/2021 по 15/01/2022. Всё это говорит о необходимости выработки дополнительных критериев для своевременного введения мер по недопущению распространения новой коронавирусной инфекции в 
целях уменьшения последующей заболеваемости и смертности от новой коронавирусной инфекции.

Среднее количество дней с момента принятия первых популяционно значимых ограничительных мер до пика заболеваемости было около 30-и дней для 82 регионов, включенных в это исследование. При этом количество дней с момента принятия ограничительных мер до пика заболеваемости варьировало по разным регионам Российской Федерации. В регионах Российской Федерации, в которых ограничительные меры были приняты относительно поздно, принятие ограничительных мер, в среднем, происходило после относительно длительного периода роста заболеваемости и при относительно высоком темпе роста заболеваемости, но впоследствии, количество дней с момента принятия ограничительных мер до пика заболеваемости в этих регионах было ниже, чем в среднем для 82 регионов Российской Федерации. Это связано с установлением нерабочих дней начиная с 30 октября 2021 года после указа Президента [8], которое, в совокупности с ограничительными мерами, принятыми главами/правительствами соответствующих регионов, стало причиной более короткого периода между моментом принятия ограничительных мер главами/правительствами этих регионов и моментом пика заболеваемости по сравнению с регионами, где ограничительные меры были введены раньше. Также в регионах, где первые ограничительные меры были приняты после относительно короткого периода роста заболеваемости, или при относительно низком темпе роста заболеваемости, количество дней с момента принятия ограничительных мер до пика заболеваемости было выше, чем в среднем для 82 регионов Российской Федерации. Это может быть связано с постепенным принятием дополнительных ограничительных мер в этих регионах. Например, в некоторых регионах сначала вводились меры по социальному дистанцированию в местах общественного питания и в других общественных местах; потом (через 7-10 дней) вводились требования о наличии QR кода и других подтверждений статуса заболевания/вакцинации при посещении общественных мест/мероприятий, и т.д. 
Наши результаты говорят о том, своевременное принятие ограничительных является одним из факторов, влияющих на смертность от новой коронавирусной инфекции. Дополнительные критерии для принятия мер по недопущению распространения новой коронавирусной инфекции могут способствовать уменьшению последующей заболеваемости и смертности от новой коронавирусной инфекции. Пример такого критерия - это принятие ограничительных мер после 2-х недель роста заболеваемости при достаточно высоком уровне смертности. Также, связь между введением разных ограничительных мер и длиной периода роста заболеваемости до пика заболеваемости говорит о том, что изучение влияния разных видов ограничительных мер на динамику заболеваемости может быть полезным для последующего выбора и применения соответствующих ограничительных мер.

\section{Список литературы}

[1] Стопкоронавирус.рф - Официальный интернет-ресурс для информирования населения по вопросам коронавируса (COVID-19) https://стопкоронавирус.pф/ [2] Yandex DataLens. Коронавирус: дашборд. https://datalens.yandex/7o7is1q6ikh23 [3] Федеральная служба государственной статистики Естественное движение населения Российской Федерации - 2020 г. https://gks.ru/bgd/regl/b20 106/Main.htm [4] US Centers for Disease Control and Prevention. Excess Deaths Associated with COVID-

\section{9. https://www.cdc.gov/nchs/nvss/vsrr/covid19/excess deaths.htm}

[5] Федеральная служба государственной статистики (Росстат). Число зарегистрированных умерших (оперативные данные)

\section{https://fedstat.ru/indicator/33556}

[6] Гольдштейн Э. М. 2022. Смертность, ассоциированная с эпидемиями гриппа и новой коронавирусной инфекции в Российской Федерации, июль 2013 года - октябрь 2021 года. COVID19-PREPRINTS.MICROBE.RU. https://doi.org/10.21055/preprints- 
[7] Talic S, Shah S, Wild H, Gasevic D, Maharaj A, Ademi Z, et al. Effectiveness of public health measures in reducing the incidence of covid-19, SARS-CoV-2 transmission, and covid-19 mortality: systematic review and meta-analysis. BMJ. 2021 Nov 17;375:e068302 [8] УКАЗ ПРЕЗИДЕНТА РОССИЙСКОЙ ФЕДЕРАЦИИ ОБ УСТАНОВЛЕНИИ НА ТЕРРИТОРИИ РОССИЙСКОЙ ФЕДЕРАЦИИ НЕРАБОЧИХ ДНЕЙ В ОКТЯБРЕ - НОЯБРЕ 2021 Г. http://publication.pravo.gov.ru/Document/View/0001202110200047 [9] Официальный интернет-портал правовой информации. http://publication.pravo.gov.ru/

[10] Электронный фонд правовых и нормативно-технических документов https://docs.cntd.ru/

[11] Федеральная служба государственной статистики (Росстат). Численность постоянного населения по возрасту на 1 января.

https://www.gks.ru/dbscripts/cbsd internal/DBInet.cgi?pl=2409019

[12] Marschner IC. Estimating age-specific COVID-19 fatality risk and time to death by comparing population diagnosis and death patterns: Australian data. BMC Medical Research Methodology volume 21, Article number: 126 (2021) 